\title{
Organizaciones lecheras en los Altos Sur de Jalisco: un análisis de las interacciones productivas
}

\author{
Roberto A. Vázquez-Valencia* \\ Ismael Aguilar-Benítez ${ }^{* *}$
}

Resumen: ${ }^{1}$ Con una perspectiva de redes y el concepto de confianza como elementos fundamentales, este trabajo explica las interacciones entre productores lecheros de diez organizaciones en la región Altos de Jalisco. Se muestra la importancia de las relaciones de confianza en la construcción y constancia de dichas interacciones, que influyen en la consolidación de agrupaciones. Y, con base en el análisis de una encuesta a productores y entrevistas a informantes clave, se presentan las interacciones de las organizaciones con instituciones e industria regionales.

Palabras clave: redes, interacciones productivas, relaciones de confianza, organizaciones de productores lecheros.

Abstract: This article utilizes a network approach and the concept of trust as basic elements for analyzing the interac-

\footnotetext{
* Secretaría de Desarrollo Rural (SEDER), Guadalajara, Jalisco, México. Correo electrónico: rvalencia@correo.chapingo.mx

** Profesor-investigador de El Colegio de la Frontera Norte (COLEF). Correo electrónico: iaguilar@colef.mx

${ }^{1}$ Este artículo se deriva del trabajo de investigación de la tesis Productores y organizaciones lecheras en la región Altos Sur de Jalisco: análisis de las interacciones y condiciones de producción, de Roberto Vázquez-Valencia, de la maestría en Desarrollo Regional 2006-2008, en el Colef, dirigida por Ismael Aguilar Benítez.
} 
tions between ten dairy producers in the Altos Sur region in Jalisco, Mexico. The article shows the importance of trustworthy relationships in the construction and durability of productive interactions, which encourage the consolidation of dairy associations. Additionally, we analyze interactions between dairy associations and regional institutions and industry based on key informant interviews and producer surveys.

Key words: networks, productive interactions, trustworthy relationships, dairy producer associations.

\section{Introducción}

Con el Tratado de Libre Comercio de América del Norte (TLCAN), el Estado mexicano inició un proceso de apertura hacia una economía de mercado, sin realizar un diagnóstico para detectar las necesidades del sector lechero para ser competitivo. ${ }^{2}$ Esta situación provocó una crisis de la producción de leche a mediados de la década de 1990, que perjudicó sobre todo a pequeños y medianos productores (Cervantes et al. 2002; Rodríguez y Chombo 1998). El TLCAN tuvo un efecto negativo directo en el sector, debido a la entrada vía importaciones, cupos y sobrecupos de importación- de leche en polvo y otros derivados lácteos a precios bajos, con respecto a los nacionales.

Hay varios factores que explican la desventaja competitiva del sector lechero nacional frente al externo: a) carencia de subsidios y otras fuentes de abatimiento de costos, tanto de elaboración como de comercialización; b) persistencia de un dualismo estructural (moderno-exportador versus tradicional-familiar); c) un comercio especializado - dependiente sobre todo del mercado de Estados Unidos- y no intraindustrial y d) la contracción de la inversión agropecuaria extranjera directa (Mella y Mercado 2006).

\footnotetext{
${ }^{2}$ Véase anexo.
} 
Tanto el proceso de apertura no planificada como las formas nuevas del consumo de lácteos constituyen retos para que los productores mejoren su competitividad. Una premisa de este trabajo es que frente a la escasez de subsidios directos es importante para los productores generar mecanismos para agruparse, y así mejorar su competitividad en precios y calidad. Por otra parte, la necesidad de ofrecer un producto "diferenciado"3 al cliente puede ser también un incentivo para la asociación; la cual podría promover mayor diversificación y mejorar los canales de comercialización.

En general, es posible identificar dos tipos de estudios sobre producción lechera en México: uno orientado hacia el análisis técnicoeconómico de variables, que permiten detectar la rentabilidad de una organización/empresa (Castro et al. 2001); otro hacia el análisis desde una perspectiva de sistemas - productivos, agroindustriales localizados y formas de organización- (Rodríguez y Chombo 1998; Boucher 2002; Boucher y Salas 2004). Un elemento no incluido en los estudios revisados es el análisis de la interacción de los productores en las organizaciones lecheras y de éstas con las instituciones de fomento agropecuario y agroindustria, entre otros.

El presente trabajo analiza las interacciones productivas y comerciales en diez organizaciones lecheras en la región Altos Sur de Jalisco, surgidas como un posible mecanismo de respuesta a los retos que enfrentan los productores individuales. Fueron seleccionadas con base en el volumen de producción y su ubicación —en toda la región- para integrar una muestra geográficamente diversificada. Aunque se obtuvo una visión general de las interacciones productivas, se reconoce que para profundizar en la explicación de éstas hace falta un análisis histórico y de estudios de caso.

La idea de partida fue que la noción de red es relevante para analizar el efecto de las interacciones sociales en los aspectos productivos. En particular, constituye un instrumento conceptual adecuado para comprender las formas de interacción de los productores en las organizaciones del sector lechero y los factores que las propician $\mathrm{u}$ obstaculizan. Se muestra cómo las relaciones de confianza influyen

\footnotetext{
${ }^{3}$ Yogures y cremas light, bajos en grasa y azúcares, así como bajos en lactosa o deslactosados, enriquecidos y con lactobacilos, entre otros.
} 
en las interacciones internas de las agrupaciones. De manera complementaria, este trabajo describe las relaciones entre organizaciones de productores con las instituciones y con la industria regional.

Las fuentes principales de información fueron productores, representantes de organizaciones y funcionarios de instituciones relacionadas con el fomento a la actividad agropecuaria. Se realizaron entrevistas con actores clave dentro de la SEDER, la Secretaría de Agricultura, Ganadería, Desarrollo Rural, Pesca y Alimentación (SAGARPA) y el Consejo para el Fomento de la Calidad de la Leche y sus Derivados, A. C. (COFOCALEC).

El texto se organiza en cuatro apartados; en el primero se recupera una tipología de relaciones de confianza, dentro del enfoque de redes, y se describe la metodología utilizada. El segundo presenta algunas características de la región de estudio y de los actores principales en el sistema producto-leche. En el tercero se exponen los hallazgos principales de la investigación y el último incluye las conclusiones y reflexiones finales.

Interacciones en la producción lechera:

el enfoque de redes y relaciones de confianza

El concepto de redes puede abordarse desde al menos tres perspectivas; una metodológica, llamada análisis de redes sociales y dos teóricas, una enfocada a las relaciones actor-red y otra a la coordinación social (De Gortari y Santos 2003). Aun cuando las redes son mecanismos de intercambio e interrelaciones muy estudiados, no existe una definición única. John Barnes fue el primero en utilizar de forma explícita el análisis de redes, y lo define de forma muy amplia: "La imagen que tengo es de un conjunto de puntos algunos de los cuales están unidos por líneas. Los puntos de la imagen son personas o a veces grupos, y las líneas indican qué individuos interactúan mutuamente" $(1954,40)$. Una limitante de esta definición, para el análisis de relaciones sociales, es que se asume una voluntad colectiva; un conjunto de interacciones en las cuales "no son las características de las personas las que importan, sino las relaciones 
que éstas mantienen entre ellas" (Granovetter 1985, 490) es decir, es una cuestión relacional.

Una perspectiva más apropiada para el presente análisis propone la posibilidad de ver una red, según Wellman (1988), como un grafo que describe un conjunto de lazos entre nodos, también llamados vértices o puntos. Esos nodos pueden ser actores o entidades sociales de cualquier otra índole, por ejemplo empresas, grupos, organizaciones, etcétera. Aquí se utilizan tres elementos importantes de la red como grafo: a) el actor, como una entidad social; pueden ser personas, asociaciones o naciones. No implica necesariamente que él actúe, sino que tiene la habilidad de comunicarse con otros de su misma clase; b) díada, la representación de los posibles vínculos entre dos actores (nodos) de una red. Su importancia radica en ser la unidad básica que se puede estudiar en una red social y c) enlaces o vínculos relacionales, pueden ser direccionales, no direccionales o recíprocos. En forma gráfica, son las líneas que conectan a unos nodos con otros, y en lo conceptual constituyen los vínculos que relacionan a los actores; pueden ser de amistad, trasferencia de recursos y conocimiento, asociación o afiliaciones y conexiones físicas, entre otros. Aquí se le llama relación al conjunto de enlaces entre actores.

Cooke, Gómez Uranga y Etxebarría (1997) introducen la confianza como un elemento relacional importante para el enfoque de redes; proponen considerar cinco en el "paradigma de redes": a) reciprocidad, entendida como buena voluntad de intercambiar información, know-how, conocimientos y bienes; b) confianza, la disposición de correr riesgo, depositando confianza en la fiabilidad de otros, c) aprendizaje, reconocimiento de que la comprensión se adquiere aprendiendo las mejores experiencias, d) asociación, como la disposición de hacer preferentemente más solida las relaciones recíprocas y e) descentralización, el reconocimiento de que la información y toma de decisión centralizados no son del todo eficientes.

Con respecto a la confianza, Sable (1993) considera que es concebida como un proceso de aprendizaje y de creación de consenso entre actores económicos con intereses comunes y competitivos. Es decir, el concepto implica una expectativa mutua de que ninguna de las partes involucradas en una transacción explotará la vulnerabilidad de los otros. 
Desde una perspectiva más apropiada para este estudio, Messner (1999) concibe a la confianza como la condición fundamental de las redes y la reciprocidad como su principio funcional. Hollinsworth y Boyer (1997) y Messner (1999) identifican a la confianza como un mecanismo de obligación con la condición funcional de las redes como algo que reduce el riesgo. Luna (2005) propone a la confianza como un componente fundamental en las interacciones relacionales colectivas enmarcadas en redes de todo tipo, debido al proceso de aprendizaje y creación de consensos. En consecuencia, la confianza es primordial para lograr la consolidación en la organización misma. Por desgracia, estas conceptuaciones carecen de elementos que permitan instrumentalizar el concepto. Una primera aproximación para hacerlo, como componente analítico, es la clasificación de Luhmann (1988) quien la define como una situación de equilibrio inestable que involucra tres aspectos: a) la confianza personal o normativa, puesta en el individuo; b) la confianza estratégica, basada en el cálculo costo/beneficio y c) la confianza técnica, basada en la reputación, el prestigio, las capacidades y recursos de los participantes.

En el campo de la confianza personal o normativa, es importante distinguir diversas clases de vínculos. En primer lugar, los basados en relaciones personales, con frecuencia reconocidas por los actores como "de amistad", aunque también pueden incluir las de parentesco; en segundo, los construidos en torno a identidades culturales, funcionales o territoriales, que no necesariamente entrañan relaciones personales y los conectados con principios o medios generalizados de comunicación (el poder legítimo, el dinero o la ley), ubicados más bien en el plano de lo denominado aquí confianza sistémica.

En cuanto a la confianza calculada o estratégica, que surge del cálculo de beneficios y costos; es decir, se origina en la expectativa de beneficio(s) mutuo(s). Está relacionada con el principio de reciprocidad y cooperación basada tanto en el intercambio como en la interdependencia de recursos. Esta confianza es lo que Haggard, Maxfield y Schneider (1997) asocian con un tipo de redes estratégicas, consideradas como maneras de cooperación para alcanzar un objetivo específico. Un problema característico de este tipo de confianza es que la heterogeneidad de los recursos de intercambio hace difícil 
los acuerdos sobre pérdidas y ganancias, y puede incrementar los costos de la negociación.

En la confianza técnica u "organizativa”, autores como Hage y Alter (1997) incluyen como determinante a la capacidad de una persona u organización de identificar los problemas, entenderlos, saber cómo y a quién recurrir para resolverlos; un aspecto fundamental que se manifiesta en el prestigio de quien la posee.

Con base en esta revisión del concepto de confianza dentro del análisis de redes, en este estudio se reportan los resultados de 27 cuestionarios aplicados a productores de leche en la región, para analizar el grado de confianza en las tres categorías descritas en las relaciones organizacionales internas. Los cuestionarios incluyeron reactivos enfocados a captar el grado de confianza de los productores hacia su representante, la respuesta se estructuró en una escala de 1-5. Este instrumento capturó también las razones de los socios para entrar en la organización. Después se modelaron matrices, mediante la aplicación NETDRAW del programa UCINET ${ }^{\circledR}$.

Para estudiar las relaciones entre organizaciones productivas e instituciones y las agrupaciones encuestadas e industria lechera regional, se realizó un análisis gráfico de redes con $\mathrm{UCINET}^{\circledR}$. Las matrices de información se generaron a partir de la aplicación de cuestionarios a diez grupos lecheros.

\section{La producción lechera en los Altos de Jalisco}

En México, - por la importancia del volumen aportado respecto al nacional- la producción lechera se concentra en cuatro cuencas: La Laguna (Coahuila y Durango), los Altos de Jalisco, Tizayuca (Hidalgo) y valle de México y las zonas aledañas al Distrito Federal (Puebla y Estado de México), así como el norte de Veracruz.

Jalisco es el productor principal de leche en el país desde 1985 (Cervantes et al. 2002). Un porcentaje relativamente alto de la población estatal (24.8) se dedica al sector primario. De acuerdo con datos de la Unión Ganadera Regional del Estado de Jalisco (UGRJ), el conjunto de los ganaderos (cerca de 300 mil) genera tres y medio millones de litros de leche diarios. El hato se estima en 810 mil ca- 
bezas, de las cuales 270 mil son vacas en producción y 230 mil son vaquillas, novillos y terneras de reemplazo. En el estado predomina el sistema de lechería familiar sobre el semitecnificado (Zorrilla et al. 1997). Dispone también de una planta industrial importante para lácteos, donde se procesan e industrializan más de dos millones de litros de leche al día. Las plantas principales son Lechera Guadalajara, la Pureza, Nestlé, 19 Hermanos, Lala y Alpura. Alimentadas por los centros de acopio de tipo comunitario e individual estratégicamente distribuidos en las regiones norte y sur de los Altos, que aportan 60 por ciento de la producción total del estado (SAGARPACentro de Estadística Agropecuaria, CEA 2000).

A pesar de que los Altos es conocida como una de las cuencas lecheras más importantes del país, su productividad aún es baja, de 6.5 litros/vaca/día hasta 7.5 en promedio (Cervantes et al. 2002). En México, los productores lecheros especializados obtienen hasta 31 litros/vaca/día (Rodríguez 1996). La superficie de la subregión Altos Sur es de $6677.36 \mathrm{~km}^{2}$, correspondiente a 8 por ciento del área total del estado. La producción lechera está ampliamente difundida en la región, lo que ha generado un incremento en la prestación de servicios para el sector, por ejemplo, los establecimientos que proveen alimentos balanceados y medicina veterinaria.

En 1993, alrededor de 95 por ciento de los productores comercializaba la leche "caliente" (Rodríguez y Chombo 1998), es decir, sin un proceso de enfriamiento para atenuar su alta perecibilidad; para 2001 ese porcentaje casi se había invertido, pues 93 por ciento ya lo hacía en frío (Cervantes et al. 2002). La incorporación de la red de frío es un indicador del cambio drástico en la comercialización en la década de 1990, sobre todo a partir de la apertura comercial. Sin embargo, el incremento en la calidad de la leche (medida de acuerdo a la reductasa ${ }^{4}$ ) exigida no se compensa con incrementos en el precio real pagado (Ibid.).

En 1998 se formó el laboratorio del COFOCALEC, con el propósito de mejorar la calidad y productividad en el sector; considerado como una alternativa para no depender sólo de los laboratorios de

\footnotetext{
${ }^{4}$ La reductasa es una enzima presente en la leche que determina su calidad microbiológica, y sirve como indicador para medir su grado de perecibilidad.
} 
la industria. ${ }^{5}$ Sin embargo, como se muestra en la gráfica, el aumento en calidad ofertada de leche en la región alteña no fue acompañada por un incremento en el precio. Esta situación fue una de las motivaciones para que los productores individuales se agruparan, en busca de mejorar su capacidad de negociación frente a las nuevas condiciones de la industria.

Caracterización de las organizaciones lecheras estudiadas

El motivo principal para organizarse, manifestado por la mayoría de los grupos encuestados, fue mejorar sus condiciones de comercio

\section{Figura 1}

Evolución del precio real al productor y de la reductasa, 1993-2000

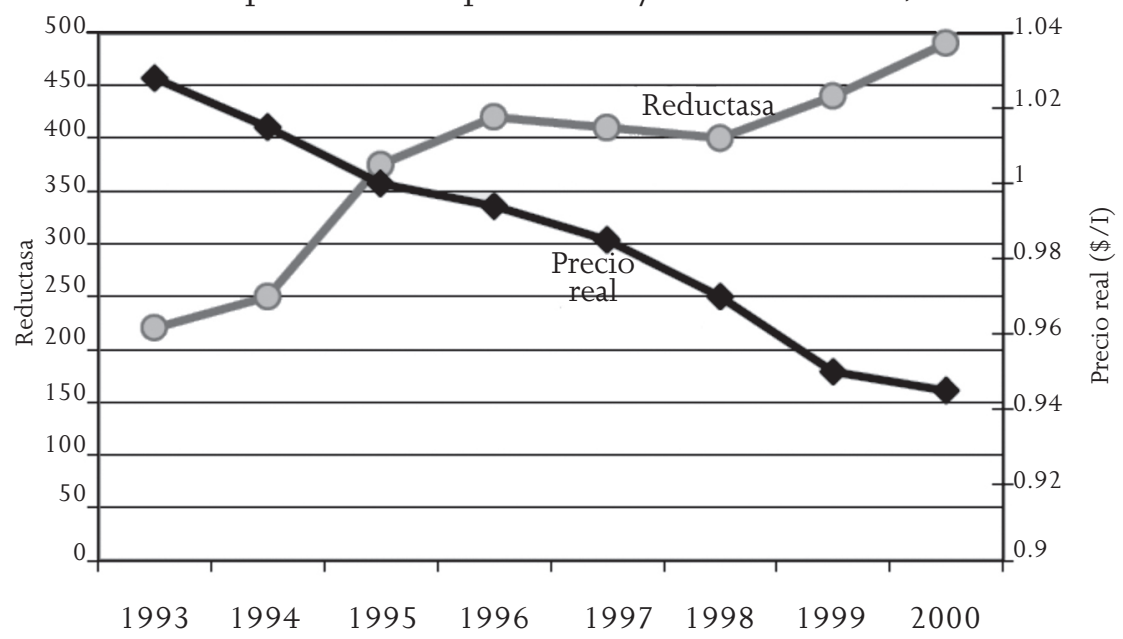

Fuente: Cervantes et al. (2002).

${ }^{5}$ COFOCALEC se constituyó en mayo de 1997 con representantes del sector público, social y privado, para atender asuntos relativos a la calidad y la productividad en el sector lechero de Jalisco. Durante 1998, y con el apoyo económico del gobierno del estado, se elaboraron los documentos requeridos por la Ley Federal sobre Metrología y Normalización y los establecidos por las normas aplicables a unidades de verificación y organismos de certificación de producto, a fin de lograr la acreditación de COFOCALEC para verificar y certificar la norma mexicana NMX-F-026-1997, Leche-Denominación, especificaciones comerciales y métodos de prueba, norma aplicable a la leche y fórmula láctea. Obtuvo el certificado de registro no. 0007 el 11 de junio de 2002, a través del cual se aprobó como Organismo Nacional de Normalización del Sistema Producto Leche; Lechera Guadalajara y Alpura son socios de él. 


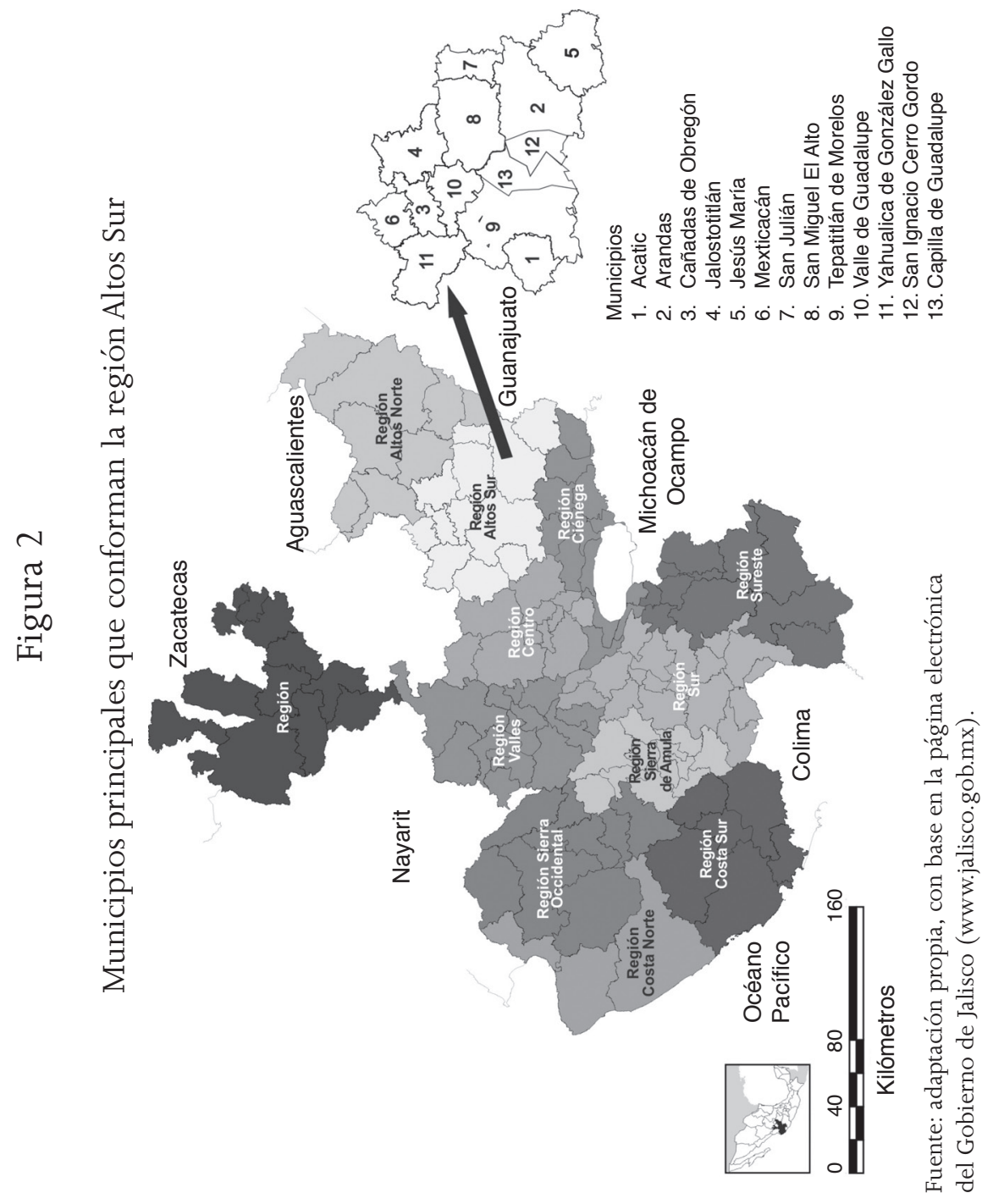


frente a los industriales de la leche (en cuanto a fijación de precio y volumen), la participación en proyectos viables para obtener recursos públicos y disminuir los costos de producción. Es decir, la organización se deriva de una exigencia competitiva de la industria: la recolección/acopio de leche para disminuir su acidificación, pues es altamente perecedera. ${ }^{6}$ Los requerimientos de calidad exigidos por la industria condujo a los productores a organizarse, para tener acceso a los recursos del Programa de Tanques Rancheros, consistente en la adquisición colectiva de tanques para el enfriamiento y venta de leche, con la finalidad de integrarse a la industria. El Gobierno de Jalisco, la SAGARPA, los Fideicomisos de Riesgo Compartido (FIRCO) y el Instituido en Relación con la Agricultura (FIRA) promovieron y apoyaron este programa entre los productores lecheros pequeños y medianos (Rodríguez y Chombo 2001). Para 2006, cerca de 90 por ciento de ellos, en los Altos Sur estaban organizados en torno a la operación de tanques para el enfriado y venta colectiva de leche (Sistema Estatal de Información para el Desarrollo Rural Sustentable para el estado de Jalisco, SEIDRUS 2006).

La figura 3 resume los rasgos principales de las diez organizaciones estudiadas en los Altos Sur de Jalisco, y a la vez se intenta caracterizar el tipo de relación entre los miembros, para formular una tipología de los grupos en la zona.

Es importante mencionar que la mayoría de estos organismos se conformó de manera formal hace alrededor de diez años, a mediados de la década de $1990 .^{7}$ Es posible afirmar que también la reconfiguración productiva es reciente, sobre todo en cuanto a la introducción de la cadena de frío, la administración colectiva del termo o centro de acopio y los recursos de apoyo obtenidos. Un elemento sobresaliente es la relación de parentesco en la mayoría de

\footnotetext{
${ }^{6}$ El proceso de incorporación de red de frío consta de cinco etapas: ordeña, transporte al centro de acopio, centro de acopio, transporte a la planta, planta procesadora. La primera es una de las más importantes, ya que después de la ordeña la leche debe enfriarse rápidamente a una temperatura de dos o cuatro grados. El transporte al centro de acopio debe realizarse lo más rápido posible, para evitar la disminución en la calidad. De igual manera, la entrega al centro de acopio debe hacerse sin demoras. Y éste a su vez debe asegurar una buena refrigeración de la leche recibida, y evitar así el crecimiento de bacterias.

${ }^{7}$ Hay que destacar que algunos grupos ya trabajaban en colectividad desde antes de su constitución formal, por ejemplo el CECOOPAL.
} 


\section{Figura 3}

Datos generales de las diez organizaciones lecheras encuestadas

\begin{tabular}{|c|c|c|c|c|}
\hline Organización* & Municipio & $\begin{array}{l}\text { Número de } \\
\text { socios }^{* *}\end{array}$ & $\begin{array}{l}\text { Fecha de } \\
\text { constitución }\end{array}$ & $\begin{array}{l}\text { Relación principal } \\
\text { de los socios }\end{array}$ \\
\hline $\begin{array}{l}\text { Centro Lechero Cooperativo } \\
\text { de los Altos de Jalisco } \\
\text { CECOOPAL (CKPA) }\end{array}$ & $\begin{array}{l}\text { Valle de } \\
\text { Guadalupe }\end{array}$ & (280) 66 & 10/octubre/1990 & $\begin{array}{l}\text { Familiar } \\
\text { Viven en la misma } \\
\text { localidad }\end{array}$ \\
\hline Prolea (PROL) & Acatic & (44) 450 & 30/agosto/1991 & Varios \\
\hline La Perla (PERL) & $\begin{array}{l}\text { San Miguel el } \\
\text { Alto }\end{array}$ & 20 & ------- & $\begin{array}{l}\text { Amistad } \\
\text { Familiares }\end{array}$ \\
\hline La Providencia (PROv) & San Julián & ------ & ------ & $\begin{array}{l}\text { Familiares } \\
\text { Viven en la misma } \\
\text { localidad }\end{array}$ \\
\hline $\begin{array}{l}\text { La Llave de Guzmán SPR de } \\
\text { RL (GUZM) }\end{array}$ & $\begin{array}{l}\text { Valle de } \\
\text { Guadalupe }\end{array}$ & 6 & $\begin{array}{l}\text { 6/noviembre } \\
\text { /2001 }\end{array}$ & $\begin{array}{l}\text { Familiares } \\
\text { Amistad }\end{array}$ \\
\hline $\begin{array}{l}\text { La Esperanza de Magueyes } \\
\text { (MAGE) }\end{array}$ & Tepatitlán & 8 & 2000 & $\begin{array}{l}\text { Familiares } \\
\text { Viven en la misma } \\
\text { localidad }\end{array}$ \\
\hline $\begin{array}{l}\text { Productos de Leche de los } \\
\text { Altos (LEAL) }\end{array}$ & $\begin{array}{l}\text { San Juan de los } \\
\text { Lagos }\end{array}$ & 4 & 1996 & $\begin{array}{l}\text { Familiares } \\
\text { Amistad }\end{array}$ \\
\hline La Palestina (PALE) & $\begin{array}{l}\text { Capilla de } \\
\text { Guadalupe }\end{array}$ & $\begin{array}{r}(300) \\
350\end{array}$ & $\begin{array}{l}\text { 8/diciembre } \\
\text { /2005 }\end{array}$ & $\begin{array}{l}\text { Amistad } \\
\text { Familiares }\end{array}$ \\
\hline Lácteos y Forrajes (LAyF) & $\begin{array}{l}\text { San José de los } \\
\text { Reynoso }\end{array}$ & 70 & 29/julio/1988 & $\begin{array}{l}\text { Amistad } \\
\text { Viven en la misma } \\
\text { localidad }\end{array}$ \\
\hline San Miguel (SUPLE) & $\begin{array}{l}\text { San Miguel el } \\
\text { Alto }\end{array}$ & 168 & $\mid-------$ & $\begin{array}{l}\text { Amistad, familiar } \\
\text { Viven en la misma } \\
\text { localidad }\end{array}$ \\
\hline
\end{tabular}
2008.

Fuente: elaboración propia, con base en fase de campo realizada en enero y febrero de

* Las siglas entre paréntesis aparecen en la etiqueta puesta para manejo, en el programa UCINET ${ }^{\circledR}$.

** El número entre paréntesis es la cantidad de socios cuando se constituyó la organización, y los que están fuera de él la cantidad actual de asociados.

las organizaciones descritas que, aunada a los criterios para identificar el avance tecnológico y el análisis de las relaciones de confianza entre los socios, permite caracterizar a cada agrupación.

La definición de la tipología de productores se basó en la propuesta del Instituto Interamericano de Cooperación para la Agricultura (IICA) (Herrera 1998), consistente en clasificarlos dentro de alguno de los niveles tecnológicos (bajo, medio y alto). Se utilizaron 
los criterios siguientes de la tipología del IICA para ubicar a las organizaciones en las categorías establecidas por la SAGARPA: a) nutricional (si más de 70 por ciento de los productores aplicaban bloques nutricionales), b) manejo reproductivo y genético (cuando más de 50 por ciento de ellos llevan registros de empadre e inseminación); c) instalaciones e infraestructura (cuando 60 por ciento contaba con corrales de manejo y ordeñadora), d) comercialización de la leche; e) tipo de ordeña (de acuerdo al porcentaje que manejaba ordeñadora manual o mecánica) y f) número de cabezas en el hato (familiar $\leq 20$, semitecnificado $>20$ y $<50$ y el tecnificado $>50$ ). A partir de dichos criterios, la categorización manejada por SAGARPA-SEDER es la siguiente: familiar o de traspatio, semitecnificada o semiintensiva y tecnificada o intensiva, incluida en la figura 4.

La mayoría de los grupos encuestados muestran tecnificación baja o media; CECOOPAL es el único que opera un establo colectivo,

Figura 4

Tipología de organizaciones lecheras en la región Altos Sur de Jalisco

\begin{tabular}{|l|c|c|c|}
\hline $\begin{array}{l}\text { Tipología de } \\
\text { organizaciones } \\
\text { lecheras }\end{array}$ & $\begin{array}{c}\text { Familiar o } \\
\text { de traspatio }\end{array}$ & $\begin{array}{l}\text { Semitecnificada } \\
\text { O semiintensiva }\end{array}$ & $\begin{array}{l}\text { Tecnificada } \\
\text { O intensiva }\end{array}$ \\
\hline CECOOPAL (CKPA) & & $\mathrm{X}$ & \\
\hline Prolea (PROL) & & $\mathrm{X}$ & \\
\hline La Perla (PERL) & $\mathrm{X}$ & $\mathrm{X}$ & \\
\hline Providencia (PROv) & $\mathrm{X}$ & & \\
\hline La Llave de Guzmán (GUZM) & $\mathrm{X}$ & & \\
\hline La Esperanza de Magueyes (MAGE) & & $\mathrm{X}$ & \\
\hline $\begin{array}{l}\text { Productores de Leche } \\
\text { de los Altos (LEAL) }\end{array}$ & $\mathrm{X}$ & & \\
\hline La Palestina (PALE) & & & \\
\hline Lácteos y Forrajes (LAyF) & & & \\
\hline San Miguel (SUPLE) & & & \\
\hline
\end{tabular}

Fuente: elaboración propia, con base en fase de campo realizada en enero y febrero de 2008. 
utiliza bloques nutricionales y cuenta con un manejo reproductivo de su hato. Estas características le ha permitido mejorar la calidad de la leche e incrementar su volumen.

Además de las organizaciones, es importante incluir en el análisis de redes a los actores institucionales, relevantes para comprender la dinámica productiva regional. En este trabajo, los actores se pueden clasificar en tres categorías: los agentes productivos, los actores institucionales y el sector público, mostradas en la figura 5.

\section{Figura 5}

Agentes productivos principales y actores institucionales relacionados con la leche

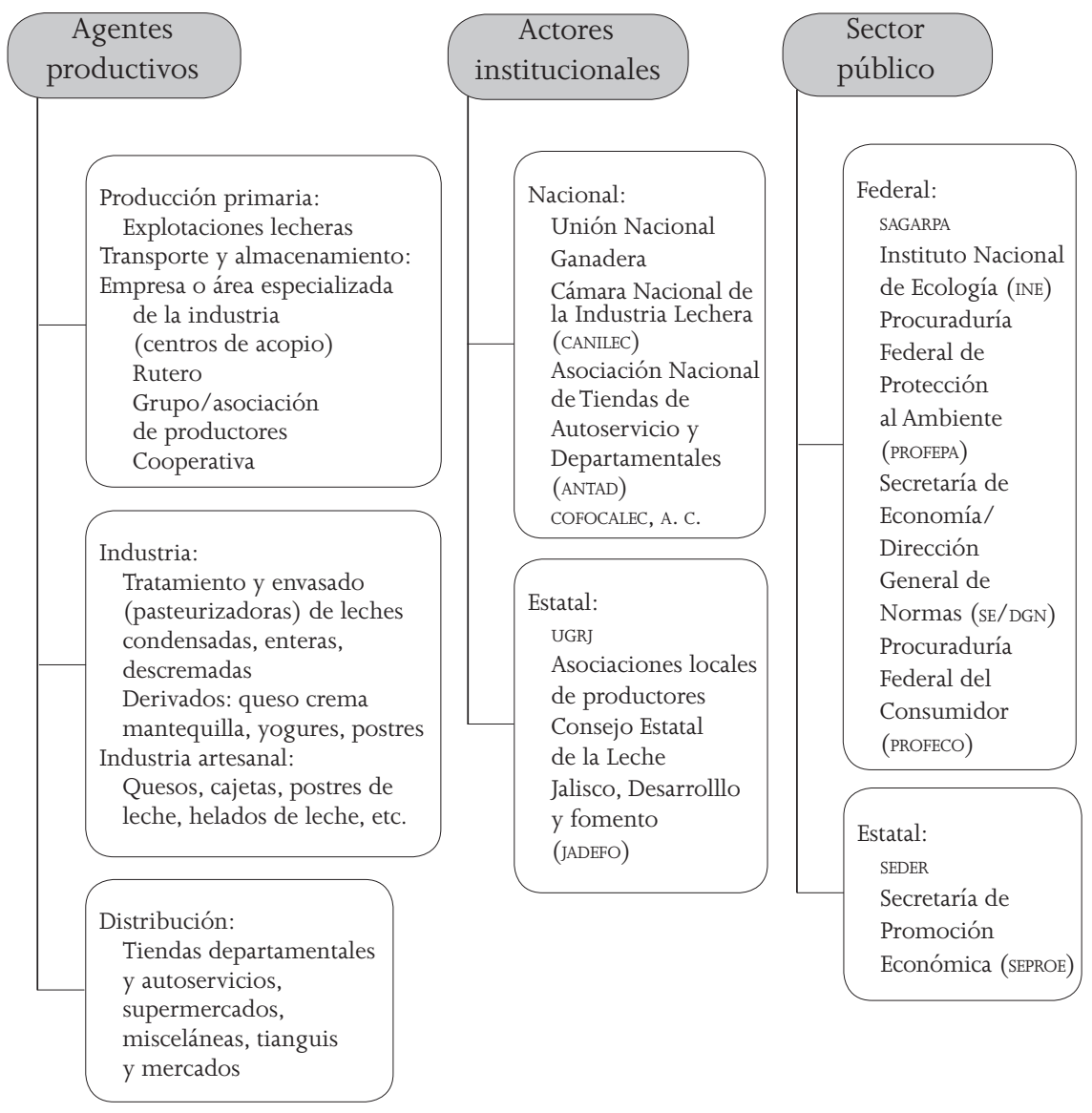

Fuente: elaboración con base en investigación directa en enero y febrero de 2008. 
Interacciones productivas: productores, organizaciones, instituciones de fomento e industria

En este apartado se incluyen los resultados de las encuestas y entrevistas realizadas a diez organizaciones lecheras de los Altos de Jalisco. En la primera parte se trata de identificar los grados de confianza manifestados por los productores hacia sus representantes e instituciones de fomento. Después se muestran las interacciones principales de los grupos con instituciones de fomento agropecuario, la industria y con los laboratorios para el análisis de la calidad del producto.

Organizaciones lecheras: la concepción de grupo

En este trabajo se asume que las organizaciones lecheras se constituyen como grupos formales, para acrecentar las capacidades de sus miembros y obtener beneficios que ayuden a elevar la productividad y competitividad, para hacer frente a las dinámicas regionales y globales en una economía de mercado. Para explorar este aspecto, se indagó en el cuestionario si los productores piensan que existen ventajas por estar asociados; siete de diez dijeron que sí, pues favorece la compra de insumos, así como la obtención del apoyo de las instituciones de fomento agropecuario y mejores precios de comercialización.

Quienes no estaban convencidos de las ventajas de pertenecer a un grupo argumentaron que eran más los costos en tiempo y trabajo que los beneficios económicos que pudieran recibir de la organización misma; así como de los apoyos y subvenciones de las instituciones de fomento agropecuario.

La negociación de las organizaciones con la industria es asimétrica (Rodríguez y Chombo 1998; Cervantes et al. 2002), y representa un elemento de "poder" a favor de la industria lechera, para fijar las reglas de la relación informal pactada, donde la industria obtiene la condición más ventajosa con el objetivo de lograr mayor calidad en el producto recibido, y ser competitiva en la elaboración de derivados lácteos. Ante una dinámica asimétrica, varios repre- 
sentantes de los organismos lecheros han optado por cumplir con las exigencias impuestas por la industria, más que por representar los intereses de sus agremiados. En este sentido, se asemejan más a subsidiarias de la industria que a asociaciones institucionalizadas con dominio propio en sus acciones. Este hecho se refleja en que seis de los diez encuestados manifiestaron inconformidad en las formas de negociación que sus representantes llevan a cabo con la industria. Algunas de las respuestas coincidieron en que, aunque la organización asegura la venta de su producto, la toma de decisiones dentro de ella es compleja:

Luis: En veces [sic] lidiamos entre nosotros como socios pero ahora podemos tener segura la venta [de leche] y no andamos batallando buscando dónde colocarla.

Pedro: $[\ldots]$ Realmente es un problemón cuando se tiene que poner uno de acuerdo [...] ya sea una mejora en las instalaciones donde está el tanque [...] o si se va a conseguir ensilado para el ganando de los socios en el tiempo de secas $[\ldots]$.

Las relaciones de confianza dentro de las organizaciones

En la encuesta se trató de captar la percepción del miembro de una asociación respecto al desempeño de su representante; ésta se clasifica en relaciones de confianza de tres tipos: personal, estratégica y técnica; establecidas y fortalecidas entre los productores, y que son elementos centrales que potencialmente sustentan un mejor funcionamiento de la organización misma. A pesar de que el intento de categorización de las relaciones de confianza puede resultar un tanto subjetivo, esto es necesario como una aproximación a la realidad compleja manifestada por la percepción de los productores lecheros. En la figura 6 se presenta la sistematización de los tres tipos de confianza, para ello se clasificó a cada organización en tres niveles por cada categoría. Esta escala se construyó con base en el análisis de los grados de confianza expresados por los entrevistados hacia sus representantes, en escala de uno a cinco. 
- Confianza personal/normativa

En la figura 6 se aprecia que los grados de confianza personal son medio o bajo. En apariencia esta información es contradictoria, por el hecho de que la mayoría de las organizaciones son familiares, y se esperaría que este tipo de confianza fuera el más elevado en relación con los otros dos (estratégica y técnica). Una explicación posible es el individualismo de los productores, quienes están acostumbrados a trabajar en su rancho y por lo general empleando a los miembros de la familia, más que en forma colectiva (Cervantes et al. 2002).

\section{- Confianza estratégica}

Cuatro de los grupos registraron un grado "alto" de confianza estratégica; sólo uno reportó uno medio y cinco fueron bajos. En la mitad de las organizaciones, los socios mostraron tener confianza estratégica en sus representantes, basada en la percepción de los beneficios que les representa producir o vender en grupo, derivados de los precios pactados con la industria. Los grados de confianza estratégica bajos se encontraron en las organizaciones que, de acuerdo con la información de los entrevistados, han tenido mayor rotación de representantes. Se puede asumir que ésta ha perjudicado las negociaciones con la industria.

\section{- Confianza técnica}

Sólo dos de los diez grupos registraron un grado "alto" de confianza técnica hacia el representante; mientras que tres revelaron uno "medio", y los cinco restantes uno bajo. Este dato sugiere que, por diversas razones, la mayoría de los socios no percibe que puede confiar en las habilidades técnicas de sus representantes para conducir a la organización a la que pertenece.

La figura 6 muestra que los grupos CECOOPAL, un establo colectivo y Prolea, una cooperativa, tuvieron la confianza más elevada en sus representantes; de media a alta. Quizá esto se relacione con los logros en la obtención de apoyos de instituciones de fomento agrope- 
cuario y por los servicios que proporciona a sus asociados. Prolea, en particular, se ha caracterizado por ser incluyente para todos los miembros, lo que ha generado una confianza técnica y estratégica "aceptables" hacia sus representantes. Los aspectos administrativos y gerenciales de la cooperativa se consideran adecuados para las necesidades de los productores.

En contraste, San Miguel (SUPLE) tuvo los grados más bajos en las tres categorías señaladas. Una primera argumentación explicativa acerca de dicho hallazgo es que carece de vías adecuadas de acceso a los ranchos donde se realiza el acopio, aunado a un bajo liderazgo de su representante y poca interacción dentro de su organización, es decir, no está vinculado con otros actores de la red, que le permitan mayor flujo de información de relevancia para el sector (contacto de comercializadores, apoyos crediticios y subvenciones).

En general, se puede afirmar que existe una yuxtaposición de las dimensiones de los tipos de confianza; por ejemplo, que exista una estratégica permite que la personal o normativa se complemente y fortalezca la interacción entre organizaciones. Por ejemplo CECOOPAL comparte "cierta" información con Prolea, donde se proveen de mayor certidumbre en cuanto a compras consolidadas de insumo y del precio de ventas de leche. Asimismo, en las cuestiones tecnológicas acerca de un proceso o producto veterinario o de manejo reproductivo, que puede bajar los costos, pero tener gran repercusión en la producción. ${ }^{8}$

A continuación se citan algunos ejemplos de las entrevistas, de los cuales se puede derivar la percepción de los tres tipos de confianza.

Gustavo: Realmente le tengo fe al representante ya que lo conozco de varios años y siempre ha sido derecho [sic] en la administración de la organización.

De esta aseveración se deduce la confianza personal/normativa, con la presencia de lenguajes coloquiales y relaciones socioculturales y productivas, al manifestar una confianza "interpersonal” más que

\footnotetext{
${ }^{8}$ Por ejemplo, una detección de mastitis a tiempo le reduce costos en medicamentos veterinarios, y de igual forma disminuye los que ocasionaría a la calidad del producto.
} 
en la de capacidades técnicas o la estratégica del representante; es la que se da en la relación más simple, la interpersonal.

El comentario siguiente es un ejemplo de confianza estratégica:

Franco: La verdad es que el representante siempre ha andado en esto de la leche incluso trabajó un tiempo en Sello Rojo... así que, pues sabe del negocio y eso [...] quieras o no, sí ayuda para que uno se motive y participe más en los asuntos de la organización.

Otra persona argumentó:

Ramiro: Realmente el encargado [representante legal] de la organización ha mostrado una capacidad para negociar con la industria y siempre se hace el pago a tiempo a nosotros [socios] y consigue buenos precios aún en las aguas [periodo de lluvias].

Figura 6

Tipos de relaciones de confianza dentro de las organizaciones lecheras

\begin{tabular}{|c|c|c|c|}
\hline $\begin{array}{c}\text { Tipos de relaciones } \\
\text { de confianza } \\
\text { Organización }\end{array}$ & $\begin{array}{c}\text { Personal/ } \\
\text { normativa }\end{array}$ & Técnica & Estratégica \\
\hline CECOOPAL (CKPA) & Media & Alta & Alta \\
\hline Prolea (PROL) & Media & Media & Alta \\
\hline La Perla (PERL) & Media & Baja & Baja \\
\hline La Providencia (PROv) & Media & Baja & Media \\
\hline La Llave de Guzmán (GUZM) & Baja & Media & Baja \\
\hline La Esperanza de Magueyes (MAGE) & Media & Baja & Baja \\
\hline Productos de Leche de los Altos (LEAL) & Media & Alta & Alta \\
\hline La Palestina (PALE) & Media & Baja & Baja \\
\hline Lácteos y Forrajes (LAy F) & Media & Media & Alta \\
\hline San Miguel (SUPLE) & Baja & Baja & Baja \\
\hline
\end{tabular}

Fuente: elaboración propia, con base en trabajo de campo de enero y febrero de 2008. Categorías asignadas por valor: baja $=1-2 ;$ media $=2-4$ y alta $=4-5$. 
Del testimonio anterior es posible inferir una confianza técnica u organizativa, ya que alude a la capacidad de una persona/organización para identificar problemas, entenderlos y saber cómo resolverlos o a quién recurrir para lograrlo. En el argumento, el productor muestra su confianza hacia el representante por la capacidad para negociar.

Interacción entre instituciones

de fomento agropecuario, agroindustria y laboratorios

Definir los niveles de interacción entre organizaciones e instituciones de fomento permite identificar su grado de integración, y la pertinencia de los apoyos ofrecidos para las necesidades reales de los productores. Por otro lado, la interacción con la agroindustria regional es relevante para conocer el grado de integración de la producción lechera y su posible dependencia de unas cuantas empresas; lo cual perjudica las posibilidades de mejora que podría generar la agrupación de productores. La probabilidad de una determinación arbitraria de la calidad hace que las organizaciones y sus miembros no confíen plenamente en los análisis obtenidos en los laboratorios de la industria. Analizar la interacción de las organizaciones con los laboratorios disponibles muestra su grado de autonomía para negociar mejores precios con la industria.

Organizaciones e instituciones de fomento

En la figura 7, los nodos definidos con las siglas FIRA, FIRCO, SAGARPA, SEDER, SEPROE Y OTROS representan a las instituciones correspondientes de fomento agropecuario que interactúan con los grupos lecheros. Las interacciones señaladas en la figura 2 con líneas (diadas) entre cada institución de fomento agropecuario con los grupos se consideran recíprocas, y se expresan con líneas de direccionalidad doble que indican la reciprocidad observada en la medida en que las relaciones institución-organización e institución-productor son retroalimentadas por la forma y seguimiento que se proporciona al recurso "liberado", para apoyo a productores/organizaciones lecheras. ${ }^{9}$

\footnotetext{
${ }^{9}$ Entendida como la disponibilidad a intercambiar información y conocimientos.
} 


\section{Figura 7}

Interacción de las organizaciones lecheras con instituciones de fomento agropecuario

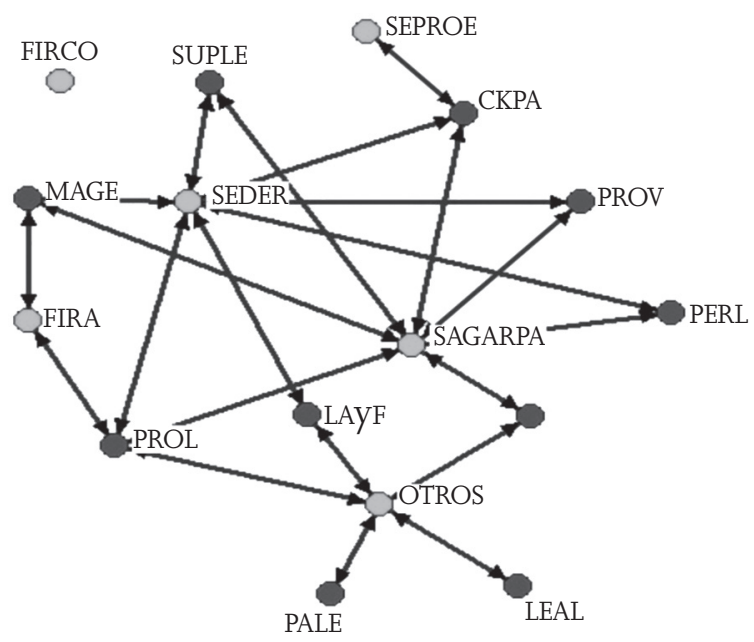

Fuente: elaboración propia, con el programa UCINET $6^{\circledR}$ para Windows ${ }^{\circledR}$, con base en encuestas y entrevistas realizadas en enero y febrero de 2008.

Las instituciones de fomento que muestran un mayor grado de centralidad — definido por el número de organizaciones que declaran tener interacciones con esas instancias- son la SAGARPA y la SEDER, que es posible definir, de acuerdo a la teoría de redes, como actores vinculadores dentro del sistema. Este resultado puede explicarse con dos elementos fundamentales: a) la existencia de una mayor diversificación de componentes que estas secretarías pueden apoyar y b) por el tipo de ayuda que otorgan, créditos a fondo perdido y en menor medida cofinanciamientos. En contraste, las instituciones con menores interacciones son FIRA Y SEPROE. Dos aspectos que dificultan el acceso a los apoyos que proporciona el FIRA son el requisito de un aval y la tasa de interés sobre el crédito; sólo CECOOPAL ha accedido a los apoyos de la SEPROE. 
Organizaciones y la agroindustria en la región

Respecto a las interacciones comerciales, la figura 8 muestra un grado elevado de centralidad de Lechera Guadalajara, S. A. de C. V., conocida como Sello Rojo. Esta empresa concentra las relaciones de comercio con 50 por ciento de las organizaciones lecheras de los Altos Sur y acopia cerca de 34 por ciento de la leche fluida. Como dato adicional, también ha logrado colocarse, además de Jalisco, en los mercados de Aguascalientes y Colima, donde compite con marcas como San Marcos, 19 Hermanos y Lala.

\section{Figura 8}

Interacción comercial de las organizaciones lecheras ${ }^{10}$

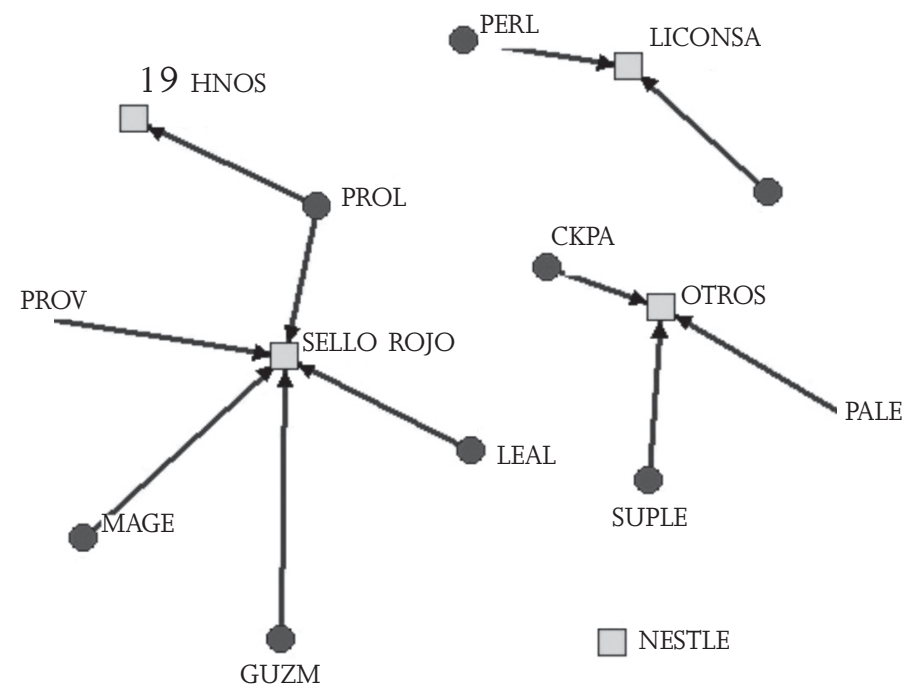

Fuente: elaboración propia, con la herramienta NETDRAW $^{\circledR}$ del programa UCINET $6{ }^{\circledR}$ para Windows ${ }^{\circledR}$, basado en encuestas y entrevistas realizadas en enero y febrero de 2008.

\footnotetext{
${ }^{10}$ Aunque Nestlé se provee de productores de la región Altos Norte, aparece aquí porque CECOOPAL le vende suero de leche. Además de jugar un papel importante en la dinámica lechera regional de los Altos. La importancia de Nestlé en los Altos Norte es mencionada por autores como Rodríguez y Chombo (1998) y Cervantes et al. (2002).
} 
Sólo Prolea tiene varios clientes: Sello Rojo y 19 Hermanos. En cambio, Lácteos y Forrajes y La Perla comercializan su producto con centros de acopio de Liconsa. En el primer caso, esta relación comercial se explica por la cercanía física, pues Liconsa es su vecino. CECOOPAL y La Palestina comercian sobre todo en el valle de México. La primera vende un mayor porcentaje (casi 60 por ciento) como derivados lácteos (quesos, yogures y cremas); la leche fluida se comercializa en la industria regional, sobre todo como suero para Nestlé.

\section{Figura 9}

Interacciones de organizaciones lecheras con laboratorios (análisis de calidad)

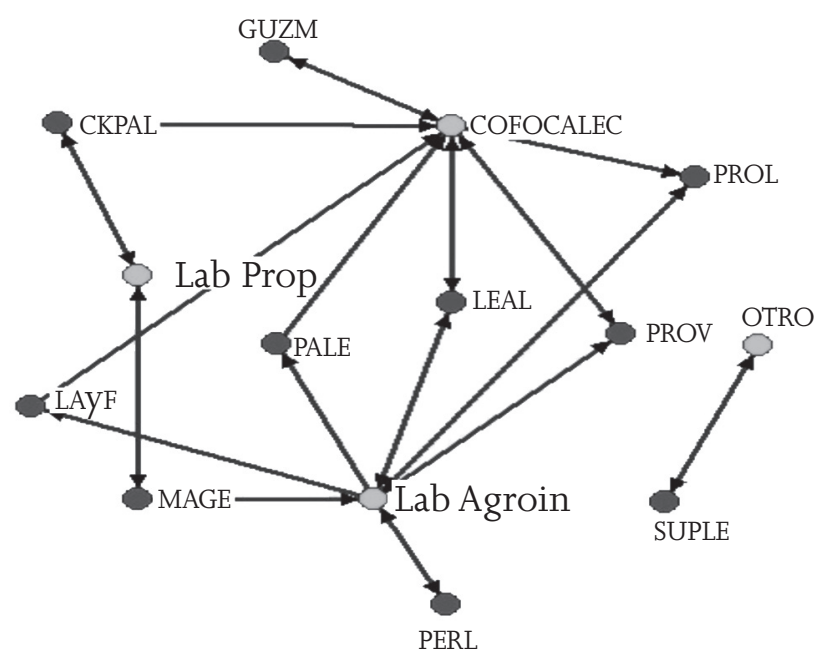

Fuente: elaboración propia, con la herramienta NETDRAW ${ }^{\circledR}$ del programa UCINET $6{ }^{\circledR}$ para Windows ${ }^{\circledR}$, basado en encuestas y entrevistas realizadas en enero y febrero de 2008.

Laboratorios y organizaciones lecheras: el criterio de calidad en el producto

Siete de las diez organizaciones estudiadas realizan sus pruebas de calidad en el laboratorio de COFOCALEC. Esto no evita que la empresa 
compradora también les exija un análisis de calidad propio. Sólo CKPAL y MAGE cuentan con sus laboratorios; Prolea y COCOOPAL realizan sus pruebas de calidad con dos laboratorios distintos, para tener mayor "margen de maniobra" en la comercialización. Estos resultados muestran que aún existe una dependencia tecnológica a favor de la industria.

\section{Conclusiones}

En este trabajo se utilizó un enfoque de redes para mostrar las interacciones productivas en los Altos Sur de Jalisco; en particular con tres tipos de confianza, relevantes para entender las relaciones de los integrantes de diez organizaciones. De acuerdo con los resultados, la estratégica es la confianza que registró el grado más alto, lo que sugiere la prioridad asignada por el productor al análisis de los costos y beneficios por pertenecer a una agrupación. Los grados de confianza técnica fueron medios y no parece existir una preocupación por mejorar las capacidades de los representantes. Paradójicamente, a pesar de que en la mayoría de las asociaciones los miembros tienen relaciones de parentesco o amistad, los grados de confianza personal son los más bajos. Los resultados sugieren que en los grupos lecheros se generan formas de cooperación-competencia, utilizadas por los miembros para enfrentar las condiciones impuestas por la industria, y aprovechar los beneficios de las instituciones de apoyo y fomento al sector. Las relaciones de confianza estratégica y técnica reflejan los frutos obtenidos de la organización. Un mayor grado de confianza técnica corresponde a mejores beneficios esperados o logrados con anterioridad.

Por otra parte, se puede argumentar que los productores no identifican como prioritaria la confianza personal, por lo que la organización se ve desde una perspectiva instrumental. Esta visión puede tener una explicación parcial, debido a la forma en que surgen los grupos, como una respuesta a las exigencias de la industria y con un criterio funcional. En general, las agrupaciones lecheras se conformaron con socios que se localizan en torno a tanques de 
enfriamiento o rutas de acopio más cercanas, sin considerar sus afinidades productivas y socioculturales. La carencia de un elemento estratégico local se refleja en la poca elaboración del producto; la gran mayoría de las organizaciones aún comercializan leche fluida, aunque ahora utilizan el proceso de enfriamiento exigido por la industria. En resumen, las lecheras de los Altos de Jalisco tienen un contenido local pobre.

Con respecto a la interacción de las agrupaciones con otros actores productivos regionales, hay elementos que confirman la baja dinámica del sistema de producción. Las instituciones de apoyo con mayor interacción con los grupos son las que permiten acceso a más diversidad de apoyos con el menor costo (créditos a fondo perdido); entonces, las organizaciones no se proponen mejorar su competitividad sino acceder a subsidios. La mayoría de ellas, y por lo tanto sus socios, depende de un solo cliente y su producción se vende dentro de la región. Puesto que una sola empresa concentra la mitad de la comercialización, existe una fuerte dependencia para la determinación de la calidad del producto; son pocas las que cuentan con laboratorios propios, y los compradores les exigen el análisis de calidad en los suyos. La capacidad de negociación del precio del producto por las organizaciones es limitada, y por lo tanto existe una dependencia comercial marcada. En este sentido, la concentración de interacciones indica también una de recursos, que paradójicamente genera una dinámica negativa; gran dependencia comercial y de trasferencia de subsidios de algunas industrias de la región.

Una posibilidad teórica es que la organización, basada en criterios instrumentales, transite hacia una forma superior en la que exista una consolidación y diversificación de las interacciones entre productores dentro de sus grupos y entre éstos y otros actores, en específico la industria y las instituciones; de manera que permita la creación de sinergias basadas en las relaciones de confianza. Así, aumentar los grados de confianza en sus tres tipos supondría una mejoría en la organización de productores, y como consecuencia en las condiciones de comercialización y productividad. Por desgracia, la evidencia encontrada no sostiene la hipótesis de un desarro- 
llo positivo; los beneficios de las interacciones productivas se concentran en algunas empresas de la industria lechera regional, que ahora recibe un producto de mejor calidad para ser competitiva. Por otra parte, los productores menos tecnificados se enfocan más a los beneficios posibles que pueden recibir de la organización por su capacidad conjunta de negociar subsidios o precios más altos, y no se interesan por las mejoras técnicas y de su propia productividad, que los haría más competitivos y les aseguraría una distribución equitativa de beneficios. Con una visión moderadamente optimista, se podría esperar que la asociación de productores permitiera la generación y mantenimiento de redes de cooperación, colaboración y competencia. Sin embargo, este estudio no detecta la existencia de estos elementos en el sector lechero de los Altos Sur. En su lugar, más que una dinámica regional propia, se observa que son las necesidades de adaptación a las exigencias de la industria para lograr mayor competitividad global las que han propiciado la agrupación de los productores. En este contexto, las empresas de la agroindustria regional y los productores más tecnificados concentran los escasos beneficios de la presión competitiva.

Recibido en agosto de 2009

Revisado en enero de 2010

\section{Bibliografía}

Barnes, J.A. 1954. Class and Comittees in a Norwegian Island Parish. Human Relations 7:39-58.

Borgatti, Steve, Martin Everett y Lin Freeman. 1999. Analytic Technologies and the University of Greenwich. UCINET 5 forWindows. Massachusetts: Natick.

Boucher, Francois. 2002. El sistema agroalimentario localizado de los productos lácteos de Cajamarca: una perspectiva para la 
agroindustria rural. Revista Sociedades Rurales, Producción y Medio Ambiente 3 (2): 1-28.

y C. I. Salas. 2004. Los desafíos de la agroindustria rural frente a la globalización. Agroindustria rural y territorio. Ponencia presentada en el Congreso internacional ARTE, Toluca, México.

Castro, L. C. J., G. Sánchez, L. F. Iruegas y G. Saucedo. 2001. Tendencias y oportunidades de desarrollo de la red leche en México. FIRA Boletín Informativo xxxiII (317). México.

Cervantes Escoto, Fernando, Adolfo Álvarez Macías y Sandra Laura Pérez Sánchez. 2002. El proceso de desintegración de grupos lecheros en la región de los Altos de Jalisco: contradicciones y tendencias. Sociedades Rurales, Producción y Medio Ambiente 3 (2): 23-37.

Cooke, P., M. Gómez Uranga y G. Etxebarria. 1997. Regional Innovation Systems: Institucional and Organizational Dimensions. Research Policy 26 (4-5): 475-491.

De Gortari, Rebeca y M. J. Santos. 2003. De contactos a redes: la construcción de redes de conocimiento a través de la formación de recursos. En Itinerarios del conocimiento: formas, dinámicas y contenido. Un enfoque de redes, coordinado por Matilde Luna. México: Instituto de Investigaciones Sociales (IIS)-Universidad Nacional Autónoma de México (UnAm)/Anthropos.

Diario Oficial de la Federación (DOF). 2004. NOM-155-SCFI-1993 (modificada en 2003 y quedó como sigue NOM-155-SCFI-2003. 3 de marzo. http://www.profeco.gob.mx/juridico/normas/noms_ infcom.asp

Granovetter, Mark S. 1985. Economic Action and Social Structure: The Problem of Embeddedness. American Journal of Sociology 91 (3): 481-510. 
78: $1360-80$.

Hage, J. y C. Alter. 1997. A Typology of Interorganizational Relationships and Networks. En Contemporary Capitalism: The Embeddedness of Institutions, compilado por R. Hollingsworth y R. Boyer, 94-126. Cambridge: Cambridge University Press.

Haggard, S., S. Maxfield y B. R. Schneider. 1997. Theories of Business and Business State-relations. En Business and the State in Developing Countries, compilado por S. Maxfield y B. R. Schneider, 36-60. Londres: Cornell University Press.

Herrera, D. 1998. Metodología para la elaboración de tipologías de actores. San José, Costa Rica: IICA.

Hollinsworth, J. Rogers y Robert Boyer. 1997. Capitalism. Institutional Economics. Organization. Cambridge: Cambridge University Press.

Luhmann, Niklas. 1988. Familiarity, Confidence, Trust: Problems and Alternatives. En Trust: Making and Breaking Cooperative Relations, compilado por Diego Gambetta, 69-92. Oxford: Basil Blackwell.

Luna, Matilde. 2005. Confianza y desempeño en las redes sociales. Revista Mexicana de Sociología 67 (1): 127-162.

Mella, José María y Alfonso Mercado. 2006. La economía agropecuaria mexicana y el tLCAN. Comercio Exterior 56 (3): 181-193.

Messner, Dirk. 1999. Del Estado céntrico a la "sociedad de redes". Nuevas exigencias a la coordinación social. En Reforma del Estado y coordinación social, coordinado por Norbert Lechner, R. Milán y F. Valdés, 77-121. México: IIs-UnAm, Plaza y Valdez.

Rodríguez Gómez, Guadalupe. 1996. Sólo es cuestión de calidad: leche y globalización en los Altos de las últimas décadas. En Los 
Altos de Jalisco al fin de siglo, coordinado por Cándido González, 29. Guadalajara: Universidad de Guadalajara.

y Patricia Chombo. 1998. Los rejuegos del poder. En Globalización y cadenas agroindustriales de la leche en Occidente, coordinado por Patricia Chombo, 328. México: Centro de Investigaciones y Estudios Superiores en Antropología Social (CIESAS); Centro de Investigación y Asistencia en Tecnología y Diseño del Estado de Jalisco; Sistema de Investigación José María Morelos; Consejo Nacional de Ciencia y Tecnología (CONACYT); Universidad Autónoma Metropolitana- Xochimilco (UAM-X).

2001. Globalización y cadenas agroindustriales de la leche en Occidente. México: CIESAS.

Sable, C. 1993. Studied Trust: Building New Forms of Cooperation in a Volatile Economy. Human Relations 46 (9): 1133-1170.

SAGARPA-CEA. 2000. Situación actual y perspectivas de la producción de leche de ganado bovino en México. México: SAGARPA-CEA, 1990-2000.

SEIDRUS. 2006. Análisis técnico económico del proceso productivo de leche en zonas específicas de Jalisco, rentabilidad de leche 2008. Estudio financiado por la Alianza para el Campo a través del programa del SEIDRUS. http://www.oeidrus-jalisco. gob.mx/

Wellman, B. 1988. Structural Analysis: from Method and Metaphor to Theory and Substance. En Social Structures: A Network Approach, editado por Barry Wellman y S.D. Berkowitz, 20. Cambridge: Cambridge University Press.

Zorrilla, R. J., V. F. Ramírez, G. G. Salazar, L. R. Martínez y R. F. J. Padilla. 1997. Descripción cualitativa y cuantitativa del sistema de lechería familiar en los Altos de Jalisco, Instituto Nacional de Investigaciones Forestales-Centro para la Investigación en Siste- 
mas Sostenibles de Producción Agropecuaria (INIFAP-CIPAV). Memorias del Seminario-taller en sistemas de producción de leche en pequeña escala. Universidad Autónoma del Estado de México, Jalisco. 


\section{Anexo}

Plazos de desgravación del sistema lechero (parte 1)

\begin{tabular}{|c|c|c|c|}
\hline \multirow[t]{2}{*}{ Fracción arancelaria } & \multirow[t]{2}{*}{ Tasa base } & \multicolumn{2}{|c|}{ Categoría de desgravación } \\
\hline & & Estados Unidos & Canadá \\
\hline \multicolumn{4}{|c|}{ Maquinaria e insumos para la producción forrajera } \\
\hline Tractores & 15 & A & A \\
\hline Arados & 10 & A & A \\
\hline Remolques & 20 & A & A \\
\hline Semillas & Exento & $\mathrm{D}$ & $\mathrm{D}$ \\
\hline Cosechadoras de forraje & 10 & A & A \\
\hline Fertilizantes $^{*}$ & 10 & $\mathrm{~B}$ & $\mathrm{~B}$ \\
\hline Agroquímicos & $10-15$ & A & A \\
\hline \multicolumn{4}{|c|}{ Ganado e insumos para la producción } \\
\hline Ganado & Exento & $\mathrm{D}$ & $\mathrm{D}$ \\
\hline Semen & Exento & $\mathrm{D}$ & $\mathrm{D}$ \\
\hline \multicolumn{4}{|c|}{ Maquinaria e insumos para la producción de alimentos } \\
\hline Maquinaria & 15 & $\mathrm{~A}$ & A \\
\hline Preparados concentrados & 10 & $\mathrm{C}$ & $\mathrm{C}$ \\
\hline Pastas oleaginosas & 15 & $\mathrm{C}$ & $\mathrm{C}$ \\
\hline Harina y pellets de alfalfa & 15 & $\mathrm{C}$ & $\mathrm{C}$ \\
\hline $\begin{array}{l}\text { Granos (sorgo) } \\
\text { a) del } 16 \text { de diciembre al } 15 \text { de mayo } \\
\text { b) del } 16 \text { de mayo al } 15 \text { de diciembre }\end{array}$ & $\begin{array}{l}\text { Exento } \\
15\end{array}$ & D & D \\
\hline Forrajes (alfalfa) & 10 & $\mathrm{C}$ & $\mathrm{C}$ \\
\hline
\end{tabular}




\section{Plazos de desgravación del sistema lechero (parte 2)}

\begin{tabular}{|l|c|c|c|}
\hline \multicolumn{1}{|c|}{ Fracción arancelaria } & Tasa base & \multicolumn{2}{c|}{$\begin{array}{c}\text { Categoría de } \\
\text { desgravación }\end{array}$} \\
\hline \multicolumn{3}{|c|}{ Maquinaria e insumos para la ordeña } \\
\hline Máquinas para la ordeña & 15 & A & A \\
\hline \multicolumn{3}{|c|}{ Maria e insumos para la industria lechera } & Canadá \\
\hline Tanques térmicos para el transporte de leche & 20 & A & A \\
\hline Maquinaria y aparatos para la industria lechera & 10 & A & A \\
\hline Leche en polvo o en pastilla & $* *$ & $* * *$ & + \\
\hline Grasa butírica & Exento & D & + \\
\hline Suero y lactosuero & 10 & C & + \\
\hline Caseína & 10 & C & + \\
\hline Leche evaporada & 20 & C & + \\
\hline Leche condensada & 15 & C & + \\
\hline \multicolumn{4}{|c|}{ Productos terminados para el consumo } \\
\hline Leche fluida envasada & 10 & C & + \\
\hline Yogur & 20 & C & + \\
\hline Mantequilla & 20 & C & + \\
\hline Queso fresco, incluido lactosuero y requesón & 40 & C & + \\
\hline Quesos maduros, rallados & 20 & C & + \\
\hline
\end{tabular}

Fuente: Cervantes et al. $(2001,64)$, con base en la norma NOM-155-SCFI-2003 (DOF 2004).

+ : productos que quedaron fuera del TLCAN entre México y Canadá.

A: desgravación inmediata. Productos que quedaron libres de arancel a partir de la entrada en vigor del TLCAN.

B: desgravación en cinco etapas anuales iguales a partir del 1 de enero de 1994. De manera que el producto en cuestión quede libre de arancel a partir del 1 de enero de 1998.

C: desgravación en 10 etapas anuales iguales a partir del 1 de enero de 1994. De forma tal que la fracción en cuestión quede libre de arancel a partir del 1 de enero de 2003.

D: productos libres de arancel cuando se llevó a cabo la negociación del TLCAN. Para productos originarios, esta situación se consolida a partir de la entrada en vigor del TLCAN.

* Incluye sulfato de amonio, superfosfatos y fosfato diamónico, el resto de los abonos y fertilizantes quedaron con una tasa base entre 10 y $15 \%$ y con categoría de desgravación A.

** Se aplicará la mayor de las tasas siguientes: 139\% ad valorem o 1160 dólares estadounidenses por tonelada métrica a desgravarse en 15 años.

*** Se aceptarán 40 mil toneladas libres de arancel, y en cantidades superiores se aplicará un arancel sobre la tasa base de 1395 para eliminarse en 15 años. 